九州大学学術情報リポジトリ

Kyushu University Institutional Repository

\title{
Elastoplastic-creep Constitutive Equation of Soils
}

Hashiguchi, Koichi

Laboratory of Agricultural Machinery, Faculty of Agriculture, Kyushu University

https://doi.org/10.5109/24208

出版情報: 九州大学大学院農学研究院紀要. 42 (1/2)，pp.211-223，1997-12. Kyushu University バージョン：

権利関係 : 


\title{
Elastoplastic-creep Constitutive Equation of Soils
}

\author{
Koichi Hashiguchi \\ Laboratory of Agricultural Machinery, Faculty of Agriculture, \\ Kyushu University 46-01, Fukuoka 812, Japan \\ (Received August 20, 1997 and accepted August 25, 1997)
}

\begin{abstract}
The elastoplastic-creep constitutive equation of soils is formulated by introducing the subloading surface model and taking the secondary consolidation characteristics into account. The mechanical response is illustrated by some numerical experiments.
\end{abstract}

\section{INTRODUCTION}

The deformation which depends on a time is called a creep in general. The secondary consolidation of soils proceeds with a time even if a stress state is fixed and thus is regarded to be a kind of the creep. The introduction of the creep due to the secondary consolidation into constitutive equations is of importance especially for the prediction of a deformation of soil structures for a long term after their constructions. It should be noted that an abrupt change of stress would not cause a plastic deformation since it takes a time for a slip of microstructure causing a plastic deformation to occur (there are no 'instant' inelastic strains, i.e. all inelastic strains require time to occur), while an elastic deformation occurs instantaneously. Therefore, it is thought that the stress goes out from the yield surface without producing a plastic deformation when it changes abruptly. The subloading surface model (Hashiguchi and Ueno, 1977, Hashiguchi, 1980, 1989, Hashiguchi et al., 1997) does not premise that the stress exists on the normal (conventional)-yield surface in a plastic loading process, introducing the subloading surface which passes always through the stress point even if the stress exists inside or outside the normal-yield surface and is similar to the normal-yield surface and letting the plastic modulus depend on the ratio of the size of the subloading surface to that of the normal-yield surface.

In this article the elastoplastic-creep constitutive equation which describes the elastoplastic deformation and the creep is formulated for soils, introducing the subloading surface model and taking the secondary consolidation characteristics into account. Besides, its mechanical response is shown by some numerical experiments.

\section{ELASTOPLASTIC-CREEP CONSTITUTIVE EQUATION WITH THE SUBLOADING SURFACE}

Let it be assumed that the stretching $\boldsymbol{D}$ (symmetric part of velocity gradient) is additively decomposed into the elastic stretching $\boldsymbol{D}^{e}$, the plastic stretching $\boldsymbol{D}^{p}$ and the creep stretching $\boldsymbol{D}^{c}$, i.e.

$$
\boldsymbol{D}=\boldsymbol{D}^{e}+\boldsymbol{D}^{p}+\boldsymbol{D}^{c},
$$


where the elastic stretching is given by

$$
\boldsymbol{D}^{e}=\boldsymbol{E}^{-1} \stackrel{\circ}{\text { \% }}
$$

$\boldsymbol{\sigma}$ is a stress and $\left({ }^{\circ}\right)$ indicates the corotational rate and the fourth-order tensor $\boldsymbol{E}$ is the elastic modulus which is given in the Hooke's type as

$$
E_{i j k l}=\left(K-\frac{2}{3} G\right) \delta_{i j} \delta_{k l}+G\left(\delta_{i k} \delta_{j l}+\delta_{i l} \delta_{j k}\right),
$$

where $K$ and $G$ are the bulk modulus and the shear modulus, respectively, which are functions of stress and internal state variables in general and $\delta_{i j}$ is the Kronecker's delta, $i$. $e . \delta_{i j}=1$ for $i=j$ and $\delta_{i j}=0$ for $i \neq j$.

Let the plastic and the creep stretchings be formulated in the following.

Consider the following yield condition as the realistic example.

$$
f(\hat{\boldsymbol{\sigma}}, \boldsymbol{H})=F(H),
$$

where

$$
\widehat{\boldsymbol{\sigma}} \equiv \stackrel{\vee}{\boldsymbol{\sigma}}-\widehat{\boldsymbol{\alpha}}
$$

$\mathscr{\boldsymbol { \sigma }}$ is the stress existing on the yield surface and $\widehat{\boldsymbol{\alpha}}$ is the kinematic hardening variable. The tensor $\boldsymbol{H}$ and the scalar $H$ denote an anisotropic and an isotropic hardening variable, respectively. Hereinafter, let it be assumed that the function $f$ has the dimension one of stress. An example of $\boldsymbol{H}$ is the rotational hardening variable of the second-order tensor (Hashiguchi, 1994).

The fact that a stress can go out from the yield surface would have to be taken into account in the elastoplastic-creep deformation process. Then, let the subloading surface (Hashiguchi and Ueno, 1977, Hashiguchi, 1980, 1989) be introduced, which always passes through the current stress $\boldsymbol{\sigma}$ and keeps the similarity to the normal-yield surface. By denoting the ratio of the size of the subloading surface to that of the normal-yield surface be denoted as $R$ and the similarity-center of the normal-yield and the subloading surfaces as $\boldsymbol{s}$, it holds that

$$
\stackrel{\vee}{\boldsymbol{\sigma}}=\frac{1}{R}\{\boldsymbol{\sigma}-(1-R) \boldsymbol{s}\} \quad(\boldsymbol{\sigma}-\boldsymbol{s}=R(\stackrel{\vee}{\boldsymbol{\sigma}}-\boldsymbol{s})),
$$

where $\mathscr{\sigma}$ on the normal-yield surface is regarded as the conjugate point of the current stress $\boldsymbol{\sigma}$ on the subloading surface.

By substituting Eq. (6) into Eq. (4), the subloading surface is described as

$$
f(\overline{\boldsymbol{\sigma}}, \boldsymbol{H})=R F(H)
$$

where

$$
\begin{gathered}
\overline{\boldsymbol{\sigma}} \equiv \boldsymbol{\sigma}-\overline{\boldsymbol{\alpha}}, \\
\overline{\boldsymbol{\alpha}} \equiv \boldsymbol{s}-R(\boldsymbol{s}-\widehat{\boldsymbol{\alpha}}) \quad(\overline{\boldsymbol{\alpha}}-\boldsymbol{s}=R(\widehat{\boldsymbol{\alpha}}-\boldsymbol{s})),
\end{gathered}
$$

where $\overline{\boldsymbol{\alpha}}$ for the subloading surface is the conjugate point of $\widehat{\boldsymbol{\alpha}}$ for the normal-yield surface.

The time-differentiation of Eq. (7) is given by

$$
\operatorname{tr}\left(\frac{\partial f(\overline{\boldsymbol{\sigma}}, \boldsymbol{H})}{\partial \boldsymbol{\sigma}} \stackrel{\circ}{\boldsymbol{\sigma}}\right)-\operatorname{tr}\left(\frac{\partial f(\overline{\boldsymbol{\sigma}}, \boldsymbol{H})}{\partial \boldsymbol{\sigma}} \stackrel{\circ}{\boldsymbol{\alpha}}\right)+\operatorname{tr}\left(\frac{\partial f(\overline{\boldsymbol{\sigma}}, \boldsymbol{H})}{\partial \boldsymbol{H}} \dot{\boldsymbol{H}}\right)=\dot{R} F+R F^{\prime} \dot{H}
$$


where $\left({ }^{\circ}\right)$ stands for the material-time derivative and

$$
F^{\prime} \equiv \frac{d F}{d H} \text {. }
$$

The evolutional rule of $R$ is given by

$$
\dot{R}=U\left\|\boldsymbol{D}^{p}\right\| \quad \text { for } \boldsymbol{D}^{p} \neq \boldsymbol{O},
$$

where $U$ is the monotonically decreasing function of $R$, satisfying

$$
\begin{aligned}
R & =0: U=+\infty, \\
0<R<1: U>0, & \\
R & =1: U=0, \\
R & >1: U<0 .
\end{aligned}
$$

Assume the associated flow rule

$$
\boldsymbol{D}^{p}=\lambda \overrightarrow{\boldsymbol{N}},
$$

where $\lambda$ is the positive proportionality factor, and the second-order tensor $\overline{\boldsymbol{N}}$ is the normalized outward-normal of the subloading surface, i.e.

$$
\overline{\boldsymbol{N}} \equiv \frac{\partial f(\overline{\boldsymbol{\sigma}}, \boldsymbol{H})}{\partial \boldsymbol{\sigma}} /\left\|\frac{\partial f(\overline{\boldsymbol{\sigma}}, \boldsymbol{H})}{\partial \boldsymbol{\sigma}}\right\|
$$

The substitution of Eqs. (12) and (14) into the consistency condition (10) leads to

where

$$
\lambda=\frac{\operatorname{tr}(\overline{\boldsymbol{N}} \dot{\boldsymbol{\sigma}})}{\bar{M}_{p}},
$$

$$
\bar{M}_{p} \equiv \operatorname{tr}(\overline{\boldsymbol{N}} \overline{\boldsymbol{a}})+\operatorname{tr}(\overline{\boldsymbol{N}} \overline{\boldsymbol{\sigma}})\left\{\frac{F^{\prime}}{F} h-\frac{1}{R F} \operatorname{tr}\left(\frac{\partial f(\overline{\boldsymbol{\sigma}}, \boldsymbol{H})}{\partial \boldsymbol{H}} \boldsymbol{h}\right)+\frac{U}{R}\right\} .
$$

$h, \boldsymbol{h}$ and $\overline{\boldsymbol{a}}$ are functions of the stress, plastic internal state variables and $\overline{\boldsymbol{N}}$ in degree one, which are related to $\dot{H}, \dot{\boldsymbol{H}}$ and $\stackrel{\circ}{\boldsymbol{\alpha}}$ as

$$
\begin{aligned}
& h \equiv \frac{\dot{H}}{\lambda}, \quad \boldsymbol{h} \equiv \frac{\stackrel{\boldsymbol{H}}{\lambda}}{\lambda}, \\
& \overline{\boldsymbol{a}} \equiv \frac{\stackrel{\check{\boldsymbol{\alpha}}}{\lambda}}{\lambda}=\boldsymbol{z}-U(\boldsymbol{s}-\hat{\boldsymbol{\alpha}})-R(\boldsymbol{z}-\hat{\boldsymbol{\alpha}}), \\
& \widehat{\boldsymbol{a}} \equiv \frac{\stackrel{\circ}{\boldsymbol{\alpha}}}{\lambda} \\
& \boldsymbol{z} \equiv \frac{\stackrel{\circ}{\boldsymbol{s}}}{\lambda}
\end{aligned}
$$

since these rate variables include $\lambda$ in degree one.

The plastic stretching is given from Eqs. (14) and (16) as

$$
\boldsymbol{D}^{p}=\frac{\operatorname{tr}\left(\overline{\boldsymbol{N}} \boldsymbol{\sigma}^{\circ}\right)}{\bar{M}_{p}} \overline{\boldsymbol{N}}
$$

which reduces to the following simple form for isotropic hardening materials with $\widehat{\boldsymbol{\alpha}}=\boldsymbol{H}=\boldsymbol{s}=\boldsymbol{O}$.

$$
\left.\begin{array}{c}
\boldsymbol{D}^{p}=\frac{\operatorname{tr}(\boldsymbol{N} \dot{\boldsymbol{\sigma}})}{M_{p}} \boldsymbol{N}, \\
\boldsymbol{N} \equiv \frac{\partial f(\boldsymbol{\sigma})}{\partial \boldsymbol{\sigma}} /\left\|\frac{\partial f(\boldsymbol{\sigma})}{\partial \boldsymbol{\sigma}}\right\|, \\
M_{p}=U F+R F^{\prime} h .
\end{array}\right\}
$$


However, by taking the fact that a plastic deformation requires time to occur into account let the plastic stretching be extended for the time-dependent deformation as follows:

$$
\boldsymbol{D}^{y}=\zeta \frac{\operatorname{tr}(\overline{\boldsymbol{N}} \dot{\boldsymbol{\sigma}})}{\bar{M}_{p}} \overline{\boldsymbol{N}}
$$

where $\zeta$ is a monotonically-decreasing function of the magnitude of stretching $\boldsymbol{D}$ satisfying the following condition.

$$
\left.\begin{array}{ll}
\zeta=1 & \text { for }\|\boldsymbol{D}\|=0 \\
\zeta \rightarrow 0 & \text { for }\|\boldsymbol{D}\| \rightarrow \infty
\end{array}\right\}
$$

An example of $\zeta$ is

$$
\zeta=\exp \left(-C_{\mathrm{t}}\|\boldsymbol{D}\|\right)
$$

$C_{\mathrm{t}}$ is the material parameter. Here, note that a stress rate-stretching relation becomes nonlinear, resulting in the rate-nonlinear constitutive equation.

Let the creep stretching $\boldsymbol{D}^{c}$ be written as

$$
\boldsymbol{D}^{c}=\boldsymbol{T}(\mathrm{t}, \boldsymbol{\sigma}, H, \boldsymbol{H}),
$$

where $\boldsymbol{T}$ is the second-order tensor function of the time t, the stress and internal state variables in general.

It holds from Eqs. (1), (2), (24) and (27) that

$$
\boldsymbol{D}=\boldsymbol{E}^{-1} \boldsymbol{\sigma}^{\circ}+\frac{\operatorname{tr}(\overline{\boldsymbol{N}} \boldsymbol{\sigma})}{\bar{M}_{p} / \zeta} \overline{\boldsymbol{N}}+\boldsymbol{T}
$$

which reduces to the following simple form for the isotropic material with $\widehat{\boldsymbol{\alpha}}=\boldsymbol{H}=\boldsymbol{s}=\boldsymbol{O}$.

$$
\boldsymbol{D}=\boldsymbol{E}^{-1} \stackrel{\circ}{\boldsymbol{\sigma}}+\frac{\operatorname{tr}(\boldsymbol{N} \stackrel{\circ}{\boldsymbol{\sigma}})}{M_{p} / \zeta} \boldsymbol{N}+\boldsymbol{T}
$$

The positive proportionality factor $\lambda$ is described in terms of the stretching $\boldsymbol{D}$ from Eq. (28) as

$$
\lambda=\frac{\operatorname{tr}((\overline{\boldsymbol{N}} \boldsymbol{E}(\boldsymbol{D}-\boldsymbol{T})\}}{\bar{M}_{p} / \zeta+\operatorname{tr}(\overline{\boldsymbol{N}} \boldsymbol{E} \overline{\boldsymbol{N}})} .
$$

The stress rate is given from Eqs. (1), (2), (24), (27) and (30) as

$$
\stackrel{\circ}{\boldsymbol{\sigma}}=\boldsymbol{E} \boldsymbol{D}-\frac{\operatorname{tr}\{(\overline{\boldsymbol{N}} \boldsymbol{E}(\boldsymbol{D}-\boldsymbol{T})\}}{\bar{M}_{p} / \zeta+\operatorname{tr}(\overline{\boldsymbol{N}} \boldsymbol{E} \overline{\boldsymbol{N}})} \boldsymbol{E} \overline{\boldsymbol{N}}-\boldsymbol{E} \boldsymbol{T} .
$$

Note that the stretching $\boldsymbol{D}$ cannot be expressed analytically by the stress rate $\boldsymbol{\sigma}^{\circ}$ since the

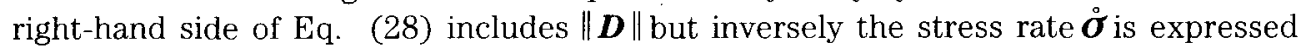
analytically by the stretching $\boldsymbol{D}$ as seen in Eq. (31).

A loading criterion is not required for the creep stretching since it proceeds always with time. On the other hand, taking the fact that $\lambda$ has to be positive, while the quantity $\bar{M}_{p} / \zeta+\operatorname{tr}(\overline{\boldsymbol{N}} \boldsymbol{E} \overline{\boldsymbol{N}})$ becomes negative in a softening process $\bar{M}_{p}<0$ for $\zeta<<1$, let the 
following loading criterion for the plastic stretching be assumed.

$$
\left.\begin{array}{c}
\boldsymbol{D}^{p} \neq \boldsymbol{O}: \frac{\operatorname{tr}\{(\overline{\boldsymbol{N}} \boldsymbol{E}(\boldsymbol{D}-\boldsymbol{T})\}}{\bar{M}_{p} / \zeta+\operatorname{tr}(\overline{\boldsymbol{N}} \boldsymbol{E} \overline{\boldsymbol{N}})}>0, \\
\boldsymbol{D}^{p}=\boldsymbol{O}: \frac{\operatorname{tr}\{(\overline{\boldsymbol{N}} \boldsymbol{E}(\boldsymbol{D}-\boldsymbol{T})\}}{\bar{M}_{p} / \zeta+\operatorname{tr}(\overline{\boldsymbol{N}} \boldsymbol{E} \overline{\boldsymbol{N}})} \leq 0 .
\end{array}\right\}
$$

\section{CONSTITUTIVE EQUATION OF SOILS}

Let the subloading surface model with the rotational hardening be introduced for the elastoplastic deformation of soils (Hashiguchi, 1994, Hashiguchi et al., 1997). The outline is described in the following.

Let it be assumed that the normal-yield surface for soils obeys the isotropic and the rotational hardenings, always passing through the origin of stress space, and thus $\widehat{\boldsymbol{\alpha}}$ exists on the origin of the stress space, i.e. $\widehat{\boldsymbol{\alpha}}=\boldsymbol{O}$. Then, the subloading surface which always passes through the current stress point and is similar to the normal-yield surface is given for soils as

$$
f(\overline{\boldsymbol{\sigma}}, \boldsymbol{H})=\bar{p}\left(1+\bar{\chi}^{2}\right)
$$

where

$$
\begin{gathered}
\bar{p} \equiv-\frac{1}{3} \operatorname{tr} \overline{\boldsymbol{\sigma}}, \overline{\boldsymbol{\sigma}}^{*} \equiv \overline{\boldsymbol{\sigma}}+\bar{p} \boldsymbol{I} \\
\overline{\boldsymbol{\eta}} \equiv \overline{\boldsymbol{Q}}-\boldsymbol{\beta}, \quad \overline{\boldsymbol{Q}} \equiv \frac{\overline{\boldsymbol{\sigma}}^{*}}{\bar{p}}, \\
\bar{\chi} \equiv \frac{\|\overline{\boldsymbol{\eta}}\|}{\bar{m}}, \\
\bar{m}=\frac{2 \sqrt{6} \sin \emptyset}{3\left\{1+a\left(1-\sin ^{2} 3 \bar{\theta}_{\sigma}\right)\right\}-\sin \emptyset \sin 3 \bar{\theta}_{\sigma}}, \\
\sin 3 \bar{\theta}_{\sigma} \equiv-\sqrt{6} \frac{\operatorname{tr} \overline{\boldsymbol{\eta}}^{3}}{\|\overline{\boldsymbol{\eta}}\|^{3}},
\end{gathered}
$$

where $\varnothing$ is the material constant. Hereinafter, the material parameter $a$ is fixed to be 0.1 for the convexity of the conical surface $\|\overrightarrow{\boldsymbol{\eta}}\|=\bar{m} . \boldsymbol{\beta}$ is the rotational hardening variable the evolutional rule of which is given as follows:

where

$$
\stackrel{\circ}{\boldsymbol{\beta}}=b_{r}\left\|\boldsymbol{D}^{v *}\right\|\|\overline{\boldsymbol{\eta}}\| \overline{\boldsymbol{\eta}}_{b},
$$

$$
\begin{gathered}
\overline{\boldsymbol{\eta}}_{b}=\bar{m}_{b} \overline{\boldsymbol{t}}-\boldsymbol{\beta}, \\
\boldsymbol{t} \equiv \frac{\overline{\boldsymbol{\eta}}}{\|\overline{\boldsymbol{\eta}}\|}, \\
m_{b}=\frac{2 \sqrt{6} \sin \emptyset_{b}}{3\left\{1+a\left(1-\sin ^{2} 3 \bar{\theta}_{\sigma}\right)\right\}-\sin \emptyset_{b} \sin 3 \bar{\theta}_{\sigma}},
\end{gathered}
$$


where $\emptyset_{b}$ is the material constant.

The evolutional rule of the similarity-center $\boldsymbol{s}$ is given as follows:

$$
\begin{aligned}
& \stackrel{\circ}{\boldsymbol{s}}=c\left\|\boldsymbol{D}^{p}\right\| \tilde{\boldsymbol{\sigma}}+\frac{1}{F}\left\{\dot{F}-\operatorname{tr}\left(\frac{\partial f\left(p_{s}, \chi_{s}\right)}{\partial \boldsymbol{\beta}} \dot{\boldsymbol{\beta}}\right)\right\} \boldsymbol{s}, \\
& f\left(p_{s}, \chi_{s}\right)=p_{s}\left(1+\chi_{S}^{2}\right),
\end{aligned}
$$

where $c$ is a material constant and

$$
\begin{aligned}
& \begin{array}{c}
\tilde{\boldsymbol{\sigma}} \equiv \boldsymbol{\sigma}-\boldsymbol{s} \\
p_{s} \equiv \frac{1}{3} \operatorname{trs}, \quad \boldsymbol{s}^{*} \equiv \boldsymbol{s}+p_{s} \boldsymbol{I},
\end{array} \\
& \boldsymbol{\eta}_{s} \equiv \boldsymbol{Q}_{s}-\boldsymbol{B}, \quad \boldsymbol{Q}_{s} \equiv \frac{\boldsymbol{s}^{*}}{\hat{p}_{s}}, \\
& \chi_{s} \equiv \frac{\left\|\boldsymbol{\eta}_{s}\right\|}{m_{s}}, \\
& m_{b}=\frac{2 \sqrt{6} \sin \varnothing}{3\left\{1+a\left(1-\sin ^{2} 3 \theta_{s}\right)\right\}-\sin \varnothing \sin 3 \theta_{s}} \text {, } \\
& \sin 3 \theta_{s} \equiv-\sqrt{6} \frac{\operatorname{tr} \boldsymbol{\eta}_{s}{ }^{3}}{\left\|\boldsymbol{\eta}_{s}\right\|^{3}} .
\end{aligned}
$$

Let the isotropic hardening/softening function be given as

$$
F=\left(F_{0}+p_{i}\right) \exp \left(\frac{H}{\rho-\gamma}\right)-p_{i},
$$

where $F_{0}$ is the initial value of $F . p_{i}(>0)$ is the material constant standing for the negative pressure in which a volume of soil becomes infinite. $\rho$ and $\gamma$ are the slope of normal-consolidation curve and the swelling curve, respectively in the space $(\ln p, \ln v)$ ( $p$ : pressure, $v$ : volume). The evolutional rule of the isotropic hardening variable $H$ is given as

where

$$
\begin{gathered}
\dot{H}=-D_{v}^{p}+D_{s}^{P}, \\
D_{v}^{P} \equiv \operatorname{tr} \boldsymbol{D}^{p}, \\
D_{S}^{P} \equiv \mu\left\|\boldsymbol{D}^{p}\right\|\left(\frac{\left\|\boldsymbol{\sigma}^{*}\right\|}{p}-m_{d}\right), \\
\frac{2 \sqrt{6} \sin \emptyset_{d}}{3\left\{1+a\left(1-\sin ^{2} 3 \theta_{\sigma}\right)\right\}-\sin \emptyset_{d} \sin 3 \theta_{\sigma}}, \\
\sin 3 \theta_{\sigma} \equiv-\sqrt{6} \frac{\operatorname{tr} \boldsymbol{\sigma}^{* 3}}{\left\|\boldsymbol{\sigma}^{*}\right\|^{3}}, \\
p \equiv-\frac{1}{3} \operatorname{tr} \boldsymbol{\sigma}, \quad \boldsymbol{\sigma}^{*} \equiv \boldsymbol{\sigma}+p \boldsymbol{I},
\end{gathered}
$$

where $\mu$ and $\emptyset_{d}$ are the material constants, while $\mu=0$ for clays and the looser the sand is, the larger $\emptyset_{d}$ is.

Let the function $U$ in the evolutional equation of $R$ be given as follows:

$$
U=-u \ln R
$$


where $u$ is the material constant.

Let the elastic bulk modulus $K$ in $\mathrm{Eq}$. (3) be given as

$$
K=\frac{p+p_{i}}{\gamma} \text {. }
$$

The elastic stretching is given from Eq. (2) with Eqs. (3) and (59) as

$$
\boldsymbol{D}^{e}=\frac{1}{3} \frac{\gamma}{p+p_{\imath}} \dot{\boldsymbol{\sigma}}_{m} \boldsymbol{I}+\frac{1}{2 G} \dot{\boldsymbol{\sigma}}^{*} .
$$

Let the creep stretching $\boldsymbol{D}^{c}$ be given as

$$
\boldsymbol{D}^{c}=\boldsymbol{T}=-\frac{1}{3} \exp \left(\xi \frac{\left\|\boldsymbol{\sigma}^{*}\right\|}{p}\right) \frac{\alpha}{\mathrm{t}}\left(\frac{\boldsymbol{\sigma}}{\sigma_{m}}\right)^{n}
$$

where $\xi, \alpha$ (coefficient of secondary consolidation) and $n$ are material constants, from which the creep volumetric stretching $D_{v}^{c}$ is given by

$$
D_{v}^{c}=-\frac{\alpha}{\mathrm{t}}
$$

in the isotropic stress state. The time-integration of Eq. (62) leads to the creep volumetric strain

$$
\varepsilon_{i}^{e}=-\alpha \ln \frac{\mathrm{t}}{\mathrm{t}_{0}}
$$

in the isotropic stress state.

Here, it should be noted that the initial time $t_{0}$ for the calculation has not to be taken to be zero but has to be interpreted as the time elapsed after the soil had began to creep in an infinite rate at $t=0$ at which soil is in the softest state.

\section{NUMERICAL EXPERIMENTS}

Calculated undrained behavior in axisymmetric compression under various deformation rates is depicted in Fig. 1, where the $D_{a}$ and $\varepsilon_{a}$ are the axial stretching and strain, respectivery, and $q=-\left(\sigma_{a}-\sigma_{i}\right)$, letting $\sigma_{a}$ and $\sigma_{l}$ denote the axial and lateral stress, respectively. The material constants and initial values are selected as follows:

material constants:

yield surface (ellipsoid) shape $\emptyset=35^{\circ}$

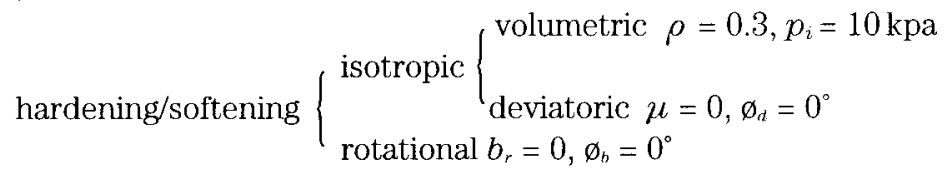

evolution of $R \quad u=100$

movement of similarity-center $c=0$

elastic constants $\gamma=0.1, \mathrm{G}=800 \mathrm{kPa}$

creep $\alpha=0.006, \xi=1.0, n=0, \mathrm{t}_{0}=150 \min \left(D_{r 0}=-4 \times 10^{-5} \mathrm{~min}^{-1}\right)$

$$
C_{\mathrm{t}}=1,000
$$

initial values:

$$
\begin{aligned}
& F_{0}=301 \mathrm{kPa}, \boldsymbol{\beta}_{0}=\boldsymbol{O}, \boldsymbol{s}_{0}=\boldsymbol{O} \mathrm{kPa} \\
& \boldsymbol{\sigma}_{0}=-300 \boldsymbol{I} \mathrm{kPa}
\end{aligned}
$$


where $\boldsymbol{\beta}_{0}$ and $\boldsymbol{s}_{0}$ and $\boldsymbol{\sigma}_{0}$ are initial values of $\boldsymbol{\beta}, \boldsymbol{s}$ and $\boldsymbol{\sigma}$, respectively. Note that inherent and induced anisotropy is ignored because of $\boldsymbol{\beta}_{0}=\boldsymbol{O}, b_{r}=0$ resulting in $\boldsymbol{\beta}=\boldsymbol{O}$ and $\boldsymbol{s}_{0}=$ $\boldsymbol{O}, c=0$ resulting in $\boldsymbol{s}=\boldsymbol{O}$.

If one keeps $q=$ const. from a certain moment on the way of the abovementioned undrained process, i.e. the undrained creep, a straight stress path with a decrease of pressure resulting in the creep rupture at the critical state line is predicted.
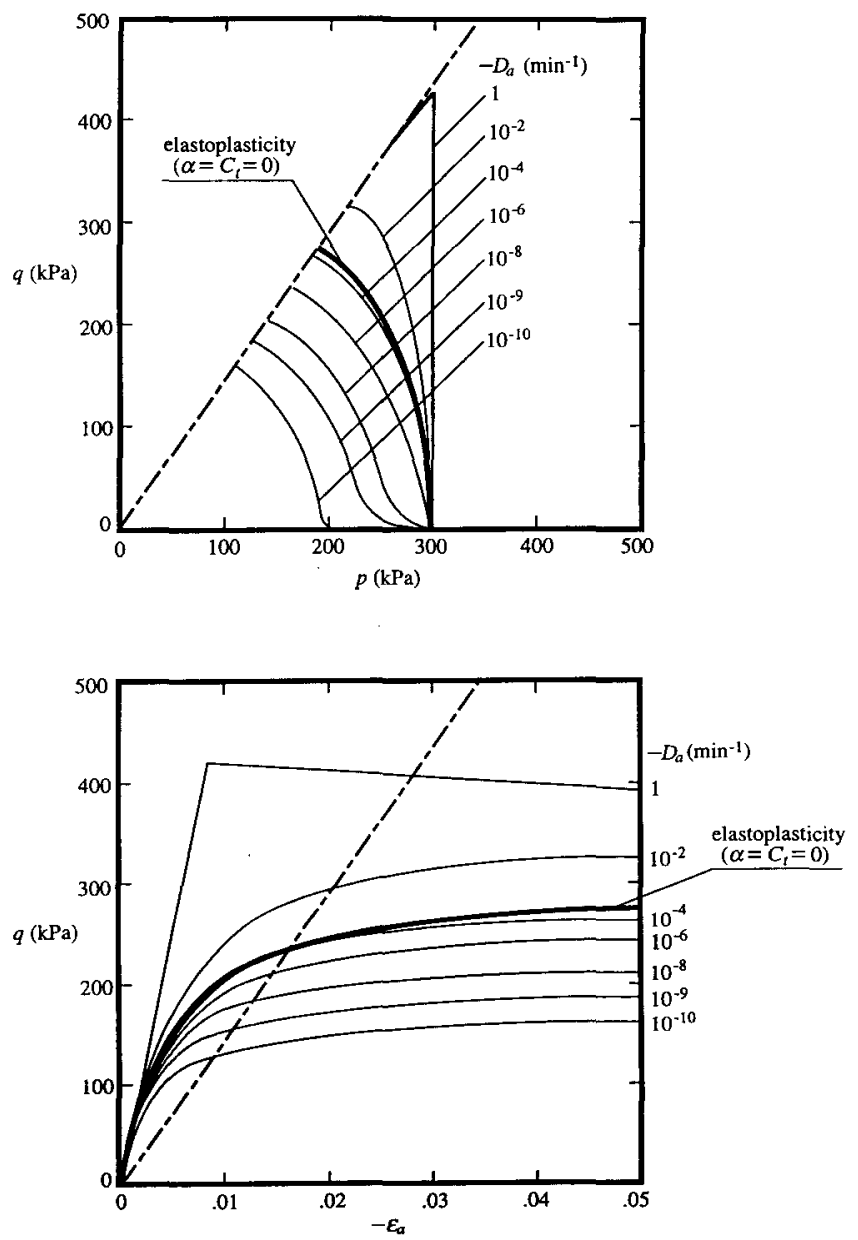

Fig. 1. The numarical experiments for undrained behavior in axisymmetric compression under various deformation speeds. 


\section{ON THE SEKIGUCHI-OHTA'S MODEL}

Sekiguchi and Ohta (1977) proposed the elasto-viscoplastic model for soils. Let the mechanical pertinence of thier model be checked in the following.

Eq. (63) is rewritten as

$$
\varepsilon_{v}^{c}=-\alpha \ln \frac{D_{v 0}^{c}}{D_{v}^{c}},
$$

using the relations $\mathrm{t}=-\alpha / D_{v}^{c}$ and $\mathbf{t}_{\mathrm{o}}=-\alpha / D_{v o}^{c}$. Further, if Eq. (64) is introduced, the volumetric strain $\varepsilon_{v}$ is written as

$$
\begin{aligned}
\varepsilon_{v} & =\varepsilon_{v}^{e}+\varepsilon_{v}^{p}+\varepsilon_{v}^{c} \\
& =f^{e}+f^{p}-\alpha \ln \frac{D_{v 0}^{c}}{D_{v}^{c}},
\end{aligned}
$$

where the functions $f^{\prime}$ and $f^{p}$ in the Cam-clay model (Schofield and Wroth, 1968) are given by

$$
\begin{aligned}
f^{e} \equiv-\frac{k}{1+e_{0}} \ln \frac{p}{p_{0}}, \\
f^{p} \equiv-\frac{\lambda-k}{1+e_{0}} \ln \frac{p}{p_{0}}-D\|\boldsymbol{\eta}\| \\
=-\frac{\lambda-k}{1+e_{0}} \ln \left\{\frac{p}{p_{0}} \exp \left(\frac{\|\boldsymbol{\eta}\|}{M}\right)\right\}, \\
\boldsymbol{\eta} \equiv \frac{\boldsymbol{\sigma}^{*}}{p}-\boldsymbol{\beta}, \\
M \equiv \frac{\lambda-k}{D\left(1+e_{0}\right)} .
\end{aligned}
$$

$D$ is the dilatancy coefficient of Shibata (1963).

Here, note that $D_{v}^{c}$ depends only on the time but the volumetric stretching $D_{v}$ depends not only on the time but also on the stress rate. That is, they differs from each other substantially. However, ignoring this fact, Sekiguchi and Ohta (1977) altered Eq. (65) as

$$
\varepsilon_{v}=f^{e}+f^{p}-\alpha \ln \frac{D_{v 0}}{D_{v}},
$$

i.e.

$$
\exp \left(\frac{-\varepsilon_{v}+f^{e}+f^{p}}{\alpha}\right) d \varepsilon_{v}=D_{w} d t
$$

without any pertinent reason, and integrating it under the initial condition $\varepsilon_{v}=f^{e}$ at $\mathrm{t}=0$, they derived the viscoplastic volumetric strain $\varepsilon_{v}^{v p}\left(\equiv \varepsilon_{v}^{p}+\varepsilon_{v}^{c}\right)$ as

$$
-\alpha \ln \left\{-D_{\imath 0} \frac{\mathrm{t}}{\alpha} \exp \left(-\frac{f^{p}}{\alpha}\right)+1\right\}=\varepsilon_{v}^{v p},
$$

i.e.

$$
p \exp \left(\frac{\|\boldsymbol{\eta}\|}{M}\right)-P_{0} \exp \left[\alpha \frac{1+e_{0}}{\lambda-k} \ln \left\{\frac{1}{-D_{v 0}} \frac{\alpha}{\mathrm{t}}\left(\exp \left(\frac{-\boldsymbol{\varepsilon}_{v}^{v p}}{\alpha}\right)-1\right)\right\}\right]=0 .
$$

Sekiguchi and Ohta (1977) regarded Eq. (71) to be the yield condition for the viscoplastic deformation of clays and further obtained the viscoplastic constitutive equation by 
substituting the associated flow rule for the viscoplastic stretching $\boldsymbol{D}^{2 p}$ into the consistency condtion of Eq. (71). The above-mentioned derivation process of the Sekiguchi-Ohta model is shown in the flowchart of Fig. 2.

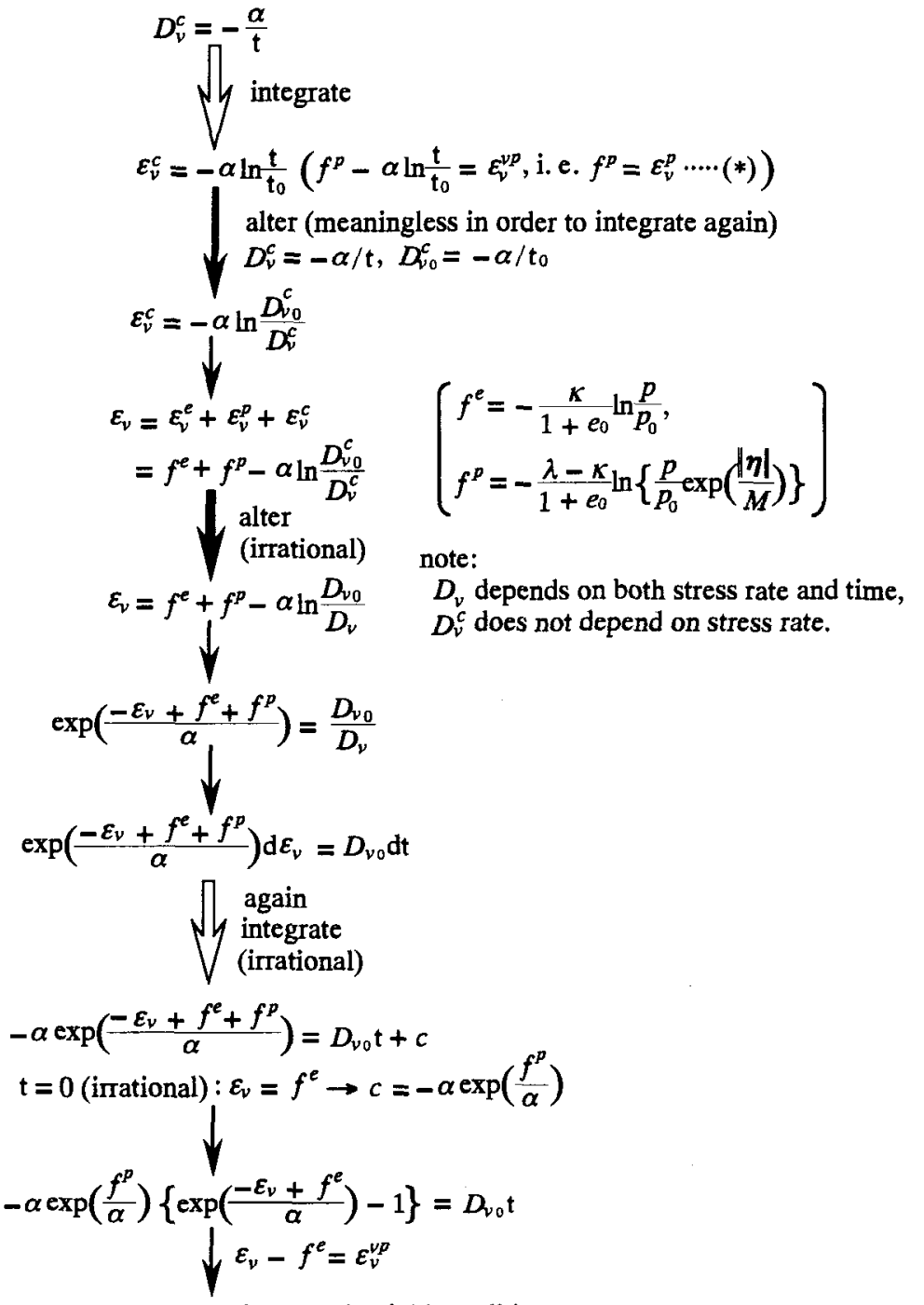

$$
\begin{aligned}
& \begin{array}{l}
-\alpha \ln \left\{1-D_{v 0} \frac{t}{\alpha} \exp \left(\frac{-f^{p}}{\alpha}\right)\right\}=\varepsilon_{v}^{v p} \\
\text { (different from } \left.f^{p}+\varepsilon_{v}^{c}=f^{p}-\alpha \ln \frac{\mathrm{t}}{\mathrm{t}_{0}}=\varepsilon_{v}^{\nu p}, \text { i. e. } f^{p}=\varepsilon_{v}^{p} \ldots . .(*) \quad ! !\right)
\end{array} \\
& \text { or } \\
& p \exp \left(\frac{|\eta|}{M}\right)-p_{0} \exp \left[\alpha \frac{1+e_{0}}{\lambda-\kappa} \ln \left\{\frac{1}{-D_{\nu 0}} \frac{\alpha}{t}\left(\exp \left(\frac{-\varepsilon_{\nu}^{v p}}{\alpha}\right)-1\right)\right\}\right]=0
\end{aligned}
$$

Fig. 2. Sekiguchi-Ohta's formulation of elasto-viscoplastic constitutive equation with various physical irrationality. 
However, note that Eq. (71) is diffrent from the following equation based on Eq. (63).

$$
\varepsilon_{v}^{v p}=f^{p}-\alpha \ln \frac{\mathrm{t}}{\mathrm{t}_{0}} \text {. }
$$

Physically meaningless but mathematically formal calculation starting from Eq. (73) without a mistake turns back to Eq. (73) itself by the vicious circle as shown in Fig. 3.

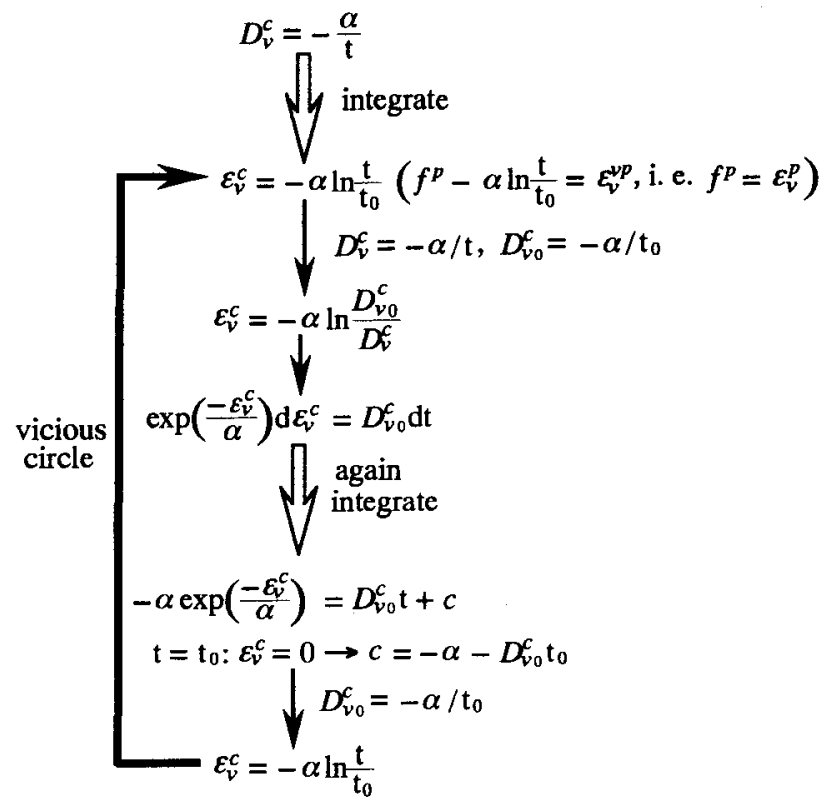

Fig. 3. Physically meaningless but mathematically formal calculation without a mistake.

The reason why Sekiguchi and Ohta (1977) got the different equation (71) is caused by the following mistakes.

1) They altered Eq. (65) into Eq. (69). However, it should be noted that the volumetric stretching $D_{v}$ and the creep volumetric stretchnig $D_{v}^{c}$ are substantially different from each other. $D_{v}$ depends not only the time but also on the stress rate, while $D_{v}^{c}$ is independent of the stress rate.

2) They integrated twice Eq. (62) to have obtained Eq. (71) in the vicious circle with the unsound alteration.

3) They put $t=0$ in the initial state of calculation. It is physically unacceptable for the creep rate to be put infinite at the initiation of calculation, while a physical property of materials is independent of calculation. As was described in the foregoing, the initial time $t_{0}$ has to be taken as the time elapsed from the softest original state, and thus $\alpha$ is to be the material constant, while $\alpha$ in the Sekiguchi and Ohta's (1977) model is not a material constant depending on when calculation is started.

Besides, it would be also physically strange that the yield function of the right-hand side in Eq. (73) or (74) includes the material parameter $\alpha$ three times. 


\section{CONCLUDING REMARKS}

The elastoplastic constitutive equation is extended so as to describe the timedenpendency of soils in this article. In this formulation the creep stretching is added to the elastic and plastic ones in the algebraical sum. Thus, it could be called the elastoplastic-creep constitutive equation. On the other hand, the viscoplastic constutive equation is formulated by modifying the plastic constitutive equation. It could be classified to the over-stress model (Perzyna, 1963a, b, 1966) and the nonstationary flow surface model (Olszak and Perzyna, 1966, 1970). The former results in the leap from the conventional plastic constitutive equation as it cannot reduce to the plastic one since a plastic deformation is not predicted when a current stress exists on the yield surface. The latter introduces a yield surface which is affected by the rate of deformation by incorporating a time. The Sekiguchi-Ohta's model in the foregoing falls in the framework of this model. However, it would not be conceivable that the yield condition is affected by the rate of deformation so remarkably as it becomes an infinite size in a moment independent of a plastic deformation. On the other hand, the elastoplastic-creep constitutive equation formulated in this article would have the simple form and the conceivable physical meaning by introducing the concept of the subloading surface which does not premise that a current stress exists on the yield surface in the plastic loading process. This concept of the extension of elastoplastic constitutive eguations so as to describe the time-dependent behavior would be applicable to materials unlimited to soils.

\section{ACKNOWLEDGEMENTS}

The author expesses his gratitude to Mr. T. Okayasu and Mr. Z.-P. Chen, the postgraduate students, Dept. Agr. Eng., Kyushu University for computer progamming of the numerical calculation.

\section{REFERENCES}

Hashiguchi, K. and Ueno, M. 1977 Elastoplastic constitutive laws of granular materials, Constitutive Equations of Soils (Proc. 9th Int. Conf. Soil Mech. Found. Eng., Spec. Session 9), Tokyo, JSSMFE, Tokyo, pp. 73-82

Hashiguchi, K. 1980 Constitutive equations of elastoplastic materials with elastic-plastic transition, $J$. Appl. Mech. (ASME), 47:266-272

Hashiguchi, K. 1989 Subloading surface model in unconventional plasticity, Int. J. Sólids Struct., 25: 917-945

Hashiguchi, K. 1994 Subloading surface model with rotational hardening, Proc. Int. Conf. Compt. Methods in Struct. \& Geotech. Eng., Hong Kong., 807-812

Hashiguchi, K., Ueno, M. and Chen, Z.-P. 1997 Elastoplastic constitutive equations of soils with the subloading surface and the rotational hardening, Int. J. Numer. Anal. Meth. Geomech., in printing.

Olszak, W. and Perzyna, P. 1966 The constitutive equations of the flow theory for a non-stationary yield condition, Proc. 11th Int. Congress of Applied Mechanics, pp. 545-553.

Olszak, W. and Perzyna, P. 1970 Stationary and nonstationary viscoplasticity, Inelastic Behavior of Solids, McGraw-Hill, pp. 53-75.

Perzyna, P. 1963a The constitutive equations for rate sensitive plastic materials, Quart. Appl. Math., 20: 321-332

Perzyna, P. 1963b The constitutive equations for workhardening and rate sensitive plastic materials, 
Proc. Vibration Problems, Warsaw, 3: 281-290

Perzyna, P. 1966 Fundamental problems in viscoplasticity, Advances in Applied Mechanics, 9: 243-377.

Sekiguchi, H. and Ohta, H. 1977 Induced anisotropy and its time dependency in clays, Constitutive Equations of Soils (Proc. Spec. Session 9, 9th Int. Conf. Soil Mech. Found. Eng.), Tokyo, pp. 229238

Schofield, A. N. and Wroth, C. P. 1968 Critical State Soil Mechanics, McGraw-Hill, London

Shibata, T. 1963 On the volumetric changes of normally-consolidated clays, Annuals, Disaster Prevention Research Institute, Kyoto University, 6: 128-134 (Japanese) 